\title{
Repeated Surgical Excision for an Unusual Variant of Nephroblastoma: Case Report and Review of the Literature
}

\author{
By J.M. Joseph, O.C. Suter, M. Nenadov-Beck, F. Gudinchet, P. Frey, and K. Meagher-Villemure \\ Lausanne, Switzerland
}

\begin{abstract}
Bilateral fetal rhabdomyomatous nephroblastoma is a rare variant of Wilms' Tumor. The authors report the evolution over 48 months of a 10-month-old baby with bilateral nephroblastoma for which a left nephrectomy was initially performed. A right kidney tumor was enucleated preserving the kidney. The transformation of the primary tumor into a completely differentiated cystic nephroblastoma or nephromalike tumor and the appearance of a metachronous lesion was seen. This report emphasizes the role of nephron-spar-
\end{abstract}

ing surgery in bilateral Wilms' Tumor when a benign transformation occurs under chemotherapy.

J Pediatr Surg 38:E13. Copyright 2003, Elsevier Science (USA). All rights reserved.

INDEX WORDS: Nephron-sparing surgery, fetal rhabdomyomatous nephroblastoma, Wilms' tumor, cystic partially differentiated nephroblastoma, multicystic nephromalike tumor.
$\mathbf{R}$ ENAL TUMORS in children represent about $8 \%$ of all pediatric malignancies. ${ }^{1}$ Primary pediatric renal tumors form a wide spectrum of histogenetically related lesions, ranging from polycystic Wilms' tumor (WT) to benign cystic nephroma, with cystic partially differentiated nephroblastoma in a midstage of differentiation. ${ }^{2}$ More than $80 \%$ of renal tumors of childhood are WT (nephroblastoma) ${ }^{1}$ A group of renal lesions resembling the metanephric blastema of the developing kidney are nephrogenic rests and nephroblastomatosis. ${ }^{3}$ All these lesions appear to be different lesions; however, they belong to the same disease spectrum. ${ }^{4}$ Indeed, the progression of nephrogenic rests to WT has been documented. ${ }^{5}$ These neoplasms appear to recapitulate histologically the embryologic development of the kidney. ${ }^{6}$ According to Beckwith et al, ${ }^{6}$ most nephrogenic rests in infants regress and do not transform into a WT.

With the advent of effective chemotherapy, the role of aggressive surgery in the management of WT has decreased over the years. The role of nephron-sparing surgery in bilateral WT appears to be considered a valid surgical option ${ }^{7}$ and is strongly considered in selected children with unilateral renal tumors. 8,9

We report the case of a 10 -month-old baby who presented with bilateral nephroblastoma of the fetal rhabdomyomatous variant with the evolution after chemotherapy, into a completely differentiated multicystic nephromalike formation. After an initial unilateral left nephrectomy, 2 subsequent lesions of the controlateral right kidney were treated successfully by enucleation. These procedures were rendered possible because a differentiation of the malignant growth to a benign tumor occurred under chemotherapy on the first lesion, and a pseudofibrous capsule had formed around the last malig- nant lesion. This report emphasizes the importance of nephron-sparing surgery in such a case.

\section{CASE REPORT}

A 10-month-old girl was examined for a febrile illness. A firm abdominal mass was palpated. On computed tomography (CT) scan examination, the mass was arising from the left kidney and involved almost entirely half of the abdomen; 2 nodules were detected in the controlateral right kidney (Fig 1a). A fetal rhabdomyomatous variant of nephroblastoma was suspected by fine-needle biopsy of the left lesion. Microscopic examination showed 2 epithelial tubular structures surrounded by mesenchymal tissue with a majority of rhabdomyoblasts. Because of the tumor size and the potential complication of a resection at that time, preoperative chemotherapy was administered according to the SIOP-93-01 protocol consisting of 4 weeks of vincristin and actinomycin $\mathrm{D}$ to reduce the volume and to render the surgical procedure more feasible.

Despite chemotherapy, the left tumor kept increasing in size, whereas the controlateral lesions remained unchanged. In view of the tumor progression, the left kidney did not appear to be salvageable anymore, and surgery became necessary despite the potential risk of damaging the surrounding organs. A left nephrectomy was performed, and macroscopic evaluation as well as a peroperative ultrasound examination was done to assess the right kidney lesions. Pathologic diagnosis was a nephroblastoma of the fetal rhabdomyomatous variant (Fig $1 \mathrm{~b}$ and d) made of blastematous component with early glomerular and tubular formations within a mesenchymal growth consisting mainly of rhabdomyoblasts which represented up to $70 \%$ of the tumor mass (Fig 1d inset). A biopsy was not performed on the right-sided lesions, and they were not resected by heminephrectomy because of

From the Departments of Pediatric Surgery, Pathology, Pediatric Oncology, and Radiology, Centre Hospitalier Universitaire Vaudois, Lausanne, Switzerland.

Address reprint requests to Jean-Marc Joseph, MD, Pediatric Surgery Department, Rue du Bugnon 19, Centre Hospitalier Universitaire Vaudois, 1011 Lausanne, Switzerland.

Copyright 2003, Elsevier Science (USA). All rights reserved. 1531-5037/03/3804-0037\$30.00/0

doi:10.1053/jpsu.2003.5015I 

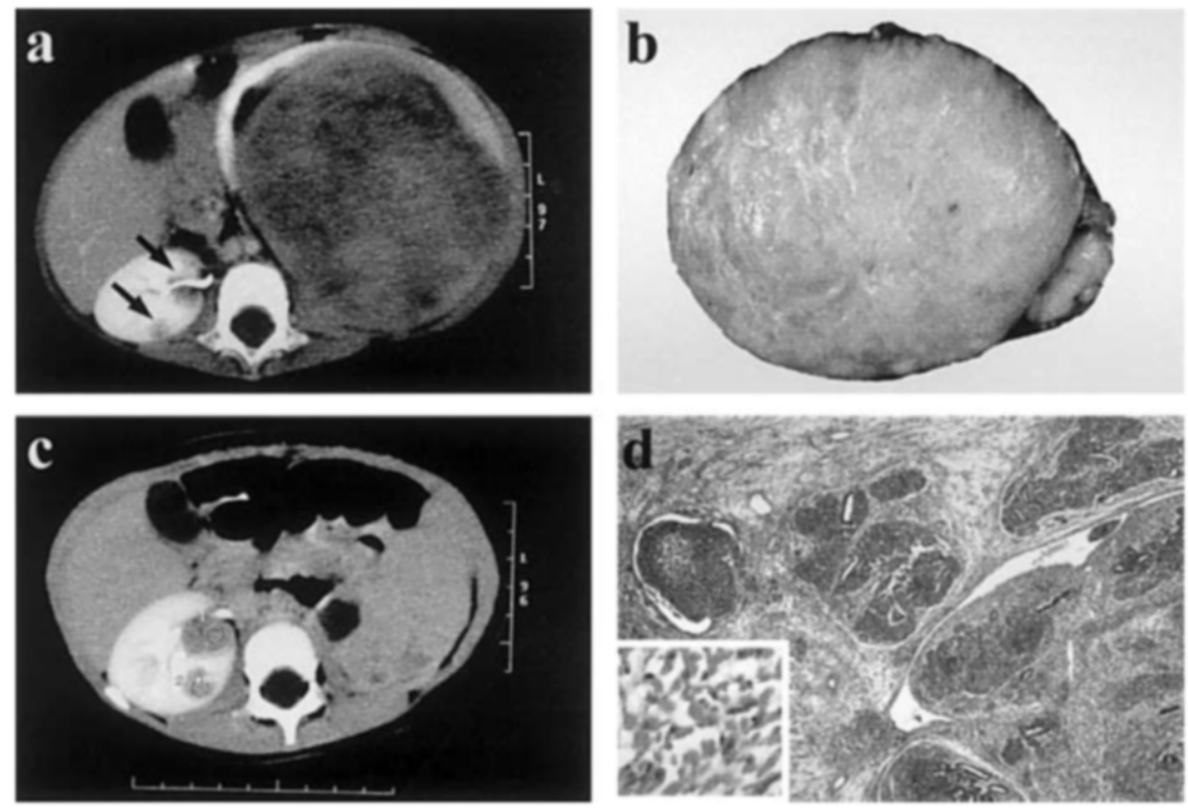

Fig 1. (a) Axial contrast-enhanced CT section at the level of the renal hilum shows a large mass almost completely replacing the left kidney. A rim of uninvolved kidney is visualized at the periphery. Two small nodules (arrows) are seen in the right kidney, one near the pelvis and one more laterally. (b) Macropathologic view of the removed mass: A large, firm, beige tumor replacing almost the whole left kidney. (c) Contrast-enhanced CT shows the enlargement of the 2 hypodense nodules in the right kidney with target circles marked 1 and $\mathbf{2}$ for the subsequent needle biopsies. The left kidney has been removed. (d) Microscopic examination of the tumor shows the triphasic pattern of the WT with blastematous, epithelial, and mesenchymatous elements. (H\&E original magnification $\times 40$; inset, focus of rhabdomyoblastic cells, original magnification $\times 400$ ). their vicinity to the renal pelvis. The right kidney was closely followed with ultrasound scan and CT scan imaging.

Thirteen months after the left nephrectomy had been done, the child was admitted emergently for a diminished general status and renal insufficiency. CT scan examination (Fig 1c) showed a right hydronephrosis caused by the enlargement of the 2 nodules compressing the pelvis. A transcutaneous pigtail catheter was inserted into the pelvis. Fine-needle biopsies of each nodule were performed. On histology, an identical cellular proliferation was found, as it had previously been found in the left kidney with blastematous and rhabdomyoblastic main components (1d). Chemotherapy consisting of vincristin, etoposid, and carboplatin, according to the high-risk group of SIOP-93-01 protocol was administered. After an initial significant tumor reduction, no further decrease could be obtained. By pursuing treatment with alternating cycles of ifosfamide and epirubicin, a surprising change could be observed on CT scan examination showing the transformation of the 2 nodules into one well-demarcated multicystic mass (Fig 2 a and b). This change motivated a right renal exploration. A tumorectomy by enucleation was performed. On macroscopic examination, the mass had a well-demarcated smooth whitish surface with, on cut section, multiple cysts of variable sizes, filled by clear fluid and separated one from the other by thin and whitish septa (Fig 2c). On histology, the cysts were lined by well-differentiated epithelial cuboidal cells, separated by fibrous bands (Fig 2d). No feature of immature or blastematous cells was found. The diagnosis of a multicystic nephromalike tumor or completely cystic differentiated nephroblastoma was made.

Five months later, during routine examination, a new tumorous nodule, located far from the previous resected one, was discovered cranially on the convexity of the kidney. A new treatment was initiated with preoperative chemotherapy consisting of vincristin, carboplatin, and doxorubicin, which induced a significant tumor reduction providing a complete tumorectomy by enucleation. On histology, the mass
Fig 2. (a) Contrast-enhanced CT scan shows enlargement of the right 2 nodules adjacent one to the other. (b) Contrast-enhanced CT scan after chemotherapy shows a hypodense multicystic lesion replacing the previous 2 nodules. (c) Macroscopic view of a cross section of the enucleated mass from the right kidney made of multiple cystic formations surrounded by firm whitish septa. (d) Microscopic examination (H\&E, original magnification $\times 40$ ) shows the cysts bordered by cuboidal epithelial cells, separated by fibrous trabeculae. No blastematous cell visualized.
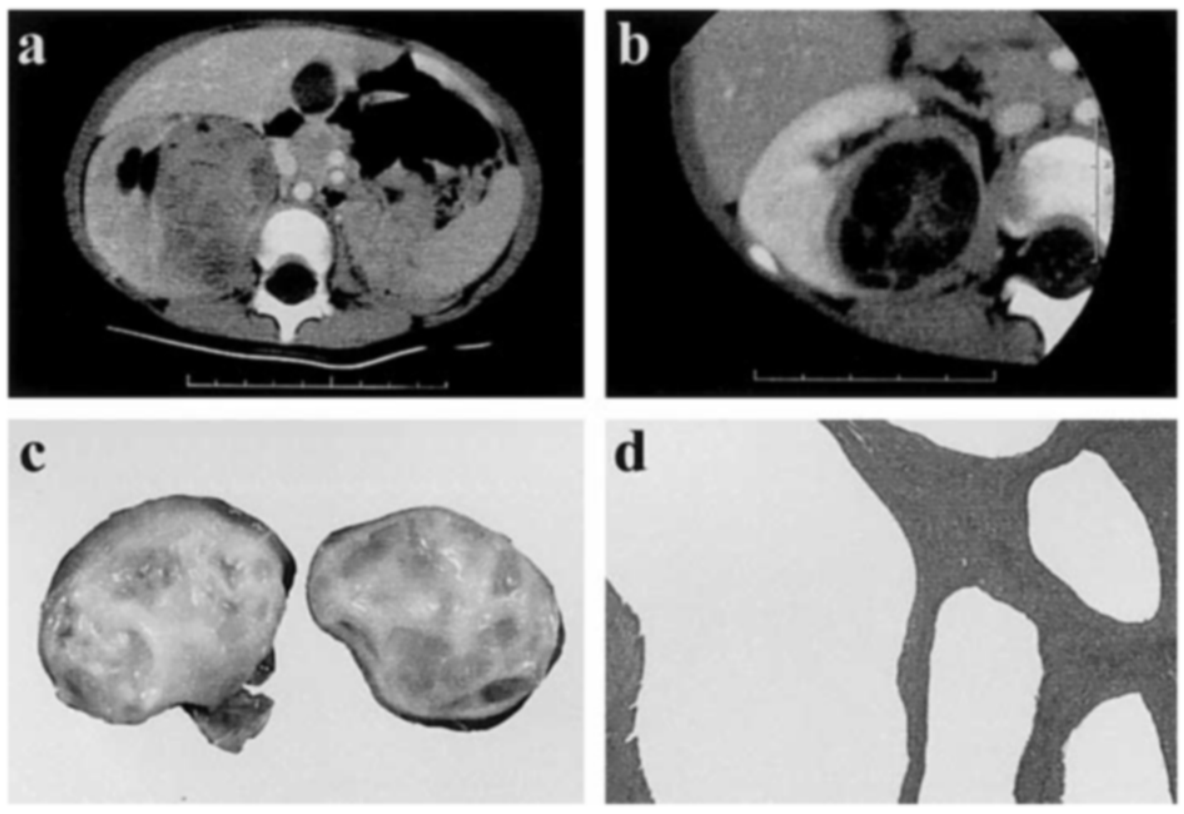
was made of a proliferation of blastematous cells with a majority of rhabdomyoblastic elements, similar to the histology seen on the previously resected left kidney. Since then, 2 years after the end of chemotherapy, the patient is disease free with adequate renal function (clearance of creatinine $85 \mathrm{~mL} / \mathrm{min} \times 1.73 \mathrm{~m}^{2}$ ).

\section{DISCUSSION}

In the majority of WT, the lesions are made of the classic triphasic combination of blastemal, stromal, and epithelial cell types. ${ }^{6}$ A rare variant is the presence of a significant rhabdomyoblastic component as part of this triphasic proliferation. For the past 30 years, the reviewed cases by the Wilms' Tumor Study group pointed out a difference between the behavior of classical WT and a stromal variant in which more than $50 \%$ of the cellular component are rhabdomyoblasts. The rhabdomyomatous fetal variant appears earlier in life with a peak under the age of 2 years; the mean age incidence of WT is 3 to 4 years. The declining number of cases after this period corresponds to the stage of development when the maturity of renal tissue decreases the chances of mutations and tumor growth. ${ }^{10}$ The rhabdomyomatous fetal variant is bilateral in $30 \%$ of the cases, whereas classical WT is bilateral only in $5 \%$ to $8 \%$. This stromal variant usually is of better prognosis.

To our knowledge, there is no documented case in which a fetal rhabdomyoblastic variant of WT had matured after chemotherapy into a completely differentiated multicystic tumor. The capacity of maturation of a WT to differentiate and transform to a cystic tumor is, however, postulated by many investigators. ${ }^{11,12}$ In our case, the histologic examination of the right kidney tumor did not show necrosis. Epithelial cysts were found separated by fibrous septa. However, because some completely mature epithelial tubules were visualized, this tumor was a labeled as a nephroma, or completely differentiated nephroblastoma, representing the final step in the differentiation of a nephroblastoma.

In our description of a bilateral fetal rhabdomyomatous nephroblastoma, as a rare variant of WT, the histologic and radiologic follow-up of the tumor evolution after surgery and chemotherapy showed a completely cystic differentiated nephroblastoma. The bilateral presentation of the tumor limited surgery at first to left nephrectomy. The imaging studies, carried out on follow-up, documented the evolution of the 2 tumor nodules on the right kidney. This radiologic procedure has the advantage of providing reliable information, compared with a surgical exploration in following the growth characteristics of dubious lesions. Serial imaging is, however, limited in identifying minute foci because it does not allow to detect the type of cellular content. Heideman et al. ${ }^{13}$ postulated that the proven efficacy of modern chemotherapy for WT, coupled with the power of imaging, allow more conservative management and help avoid the entire loss of renal function.

The role of primary exenterative renal surgery in the treatment of WT has considerably decreased with the progressive development of more effective chemotherapy. The chemoresponsiveness of many large tumors has allowed partial nephrectomy to be considered. ${ }^{1}$ However, bilateral WT develop in $5 \%$ to $8 \%$ of children with WT. Retention of renal tissue carries the potential risk of recurrence and represents a difficult management problem for surgeons and oncologists.

Many investigators advocate nephron-sparing surgery in children with bilateral WT, ${ }^{2,14}$ In a recent study, Coopper et a ${ }^{15}$ suggest that patients with bilateral WT who have a good response to initial chemotherapy and a favorable histology should have a nephron-sparing approach. The latter has a high likelihood of cure with preservation of renal function. For patients with diffuse anaplasia, however, the goal of nephron-sparing treatment should be avoided. Strong consideration should be given in this selected population to immediate radical nephrectomy or bilateral nephrectomy and renal transplantation. In children with bilateral WT and no anaplasia, a nephron-sparing approach after preoperative chemotherapy is advantageous. The survival rate using this strategy was estimated at $75 \%$ and subsequent normal renal function preservation. ${ }^{15}$ These results are in accordance with many published results of conservative surgical treatment of bilateral WT with favorable histology.7,16

This report supports the hypothesis that WT can mature under chemotherapy and can give rise to a benign multicystic tumor. It emphasises the adequacy of repeated tumoral excision instead of bilateral nephrectomy in such presentation. This approach also may be considered in case of an unilateral lesion with a high risk of metachronous tumor. However, because of the risk that some other dormant nephrogenic rests could progress and become hyperplasic or malignant, as it has been presumed in our case with the occurrence of the last resected lesion, a close follow-up is mandatory.

\section{REFERENCES}

1. D’Angio GJ, Breslow N, Beckwith JB, et al: Treatment of Wilms' tumor. Results of the Third National Wilms' Tumor Study. Cancer 64:349-360, 1989

2. Domizio P, Risdon RA: Cystic renal neoplasms of infancy and childhood: A light microscopical, lectin histochemical and immunohistochemical study. Histopathology 19:199-209, 1991
3. Beckwith JB: Precursor lesions of Wilms tumor: Clinical and biological implications. Med Pediatr Oncol 21:158-168, 1993

4. Rous SN, Bailie MD, Kaufman DB, et al: Nodular renal blastema, nephroblastomatosis, and Wilms' tumor. Different points on the same disease spectrum? Urology 8:599-604, 1976

5. Stone MM, Beaver BL, Sun CC, et al: The nephroblastomatosis 
complex and its relationship to Wilms' tumor. J Pediatr Surg 25:933937; discussion 937-938, 1990

6. Beckwith JB, Kiviat NB, Bonadio JF: Nephrogenic rests, nephroblastomatosis, and the pathogenesis of Wilms' tumor. Pediatr Pathol 10:1-36, 1990

7. Fuchs J, Wunsch L, Flemming $P$, et al: Nephron-sparing surgery in synchronous bilateral Wilms' tumors. J Pediatr Surg 34:1505-1509, 1999

8. Cozzi DA, Schiavetti A, Morini F, et al: Nephron-sparing surgery for unilateral primary renal tumor in children. J Pediatr Surg 36:362365,2001

9. Moorman-Voestermans CG, Aronson DC, Staalman CR, et al: Is partial nephrectomy appropriate treatment for unilateral Wilms' tumor? J Pediatr Surg 33:165-170, 1998

10. Charles AK, Brown KW, Berry PJ: Microdissecting the genetic events in nephrogenic rests and Wilms' tumor development. Am J Pathol 153:991-1000, 1998
11. Joshi VV, Beckwith JB: Multilocular cyst of the kidney (cystic nephroma) and cystic, partially differentiated nephroblastoma. Terminology and criteria for diagnosis. Cancer 64:466-479, 1989

12. Vujanic GM, Jenney ME, Adams H, et al: Juxtaposed cystic nephroma and Wilms' tumor. Pediatr Dev Pathol 3:91-94, 2000

13. Heideman RL, Haase GM, Foley CL, et al: Nephroblastomatosis and Wilms' tumor. Clinical experience and management of seven patients. Cancer 55:1446-1451, 1985

14. Wigger HJ: Fetal rhabdomyomatous nephroblastoma-A variant of Wilms' tumor. Hum Pathol 7:613-623, 1976

15. Cooper CS, Jaffe WI, Huff DS, et al: The role of renal salvage procedures for bilateral Wilms tumor: A 15-year review. J Urol $163: 265-268,2000$

16. Kumar R, Fitzgerald R, Breatnach F: Conservative surgical management of bilateral Wilms tumor: Results of the United Kingdom Children's Cancer Study Group. J Urol 160:1450-1458, 1998 